\title{
RESEARCH ARTICLE \\ Computational Investigation of Beryllium and Lithium Performance in Future Fusion Tokamaks
}

\author{
M. A. Bourham ${ }^{1}$ B. F. Mohamed ${ }^{2}$ N. M. Elbasha ${ }^{3 *}$ \\ 1. North Carolina State University, Department of Nuclear Engineering, Raleigh, NC 27695, USA \\ 2. Plasma Physics Department, Nuclear Research Centre, Atomic Energy Authority, Cairo, Egypt \\ 3. Physics Department, Faculty of Science, Ain Shams University, Cairo, Egypt
}

Received: 23 December 2021; Accepted: 22 January 2022; Published Online: 26 January 2022

\begin{abstract}
Low-z materials are exemplary candidates in tiling critical plasma-facing components in future fusion reactors due to their low ablation rates under intense high heat fluxes especially during abnormal and hard disruption events. Beryllium and Lithium as low-z materials show good performance as plasma-facing materials in current tokamak. Future tokamaks will exhibit long duration hard disruptions, which in turn requires further investigation of plasma-facing materials, as $\mathrm{Li}$ and $\mathrm{Be}$, to judge their performance and evaluate their erosion rates. Electrothermal plasma capillary discharges are used to simulate the high-heat flux deposition on materials to assess their erosion rates. The electrothermal plasma code ETFLOW, which is written for capillary discharges to predict the plasma parameters and erosion rates is used to simulate the high-heat flux conditions similar to expected disruption events for simulated heat fluxes from as low as $\sim 50$ to as high as $\sim 290 \mathrm{GW} / \mathrm{m}^{2}$ with a reconnoitering of generating the Be and Li plasmas up to the third ionization $\left(\mathrm{Br}^{++}, \mathrm{Li}^{+++}\right)$. Performance of $\mathrm{Be}$ and $\mathrm{Li}$ under the lowest capillary discharge currents $(50 \mathrm{kA}$ and $100 \mathrm{kA}$ ) is almost identical, however, Li shows sharper increase in the plasma pressure, heat flux, total ablated mass and the exit velocities than Be for higher discharge currents (150, 200 and $250 \mathrm{kA})$. This huge difference between the performance of $\mathrm{Li}$ and $\mathrm{Be}$ under low and high heat fluxes can be an important issue for the future magnetic fusion reactors.
\end{abstract}

Keywords: Plasma facing materials, Tokamak, Hard disruptions, The next fusion reactors, The low-z materials

\section{Introduction}

Magnetic fusion Tokamak reactors like ITER will be the first step to test the viability of fusion and to help solving the engineering problems associated with such reactors. One of the challenges in these devices is the plasma-material interactions (PMI) issues because impurities produced as a result of these interactions deteriorate plasma performance. There is also the effect on the lifetime of the plasma-facing materials (PFMs) due to ablation resulting from high heat flux exposure during normal and abnormal operational regimes. These impurities contaminate the core plasma and dilute the hydrogenic fuel, which gives rise to the loss of energy due to increased Bremsstrahlung radiation.

\footnotetext{
*Corresponding Author:

N. M. Elbasha,

Physics Department, Faculty of Science, Ain Shams University, Cairo, Egypt;

Email: nermeen_elsayed@sci.asu.edu.eg
} 
Most desirable properties of plasma-facing material are the low atomic number, low sputtering yield, high heat capacity, predictable and reliable hydrogen recycling characteristics, high melting and vaporization temperatures, and high thermal conductivity. Consequently, low $\mathrm{Z}$ materials are preferred for first wall of fusion reactors like Carbon, Beryllium and Lithium (in the reactor blanket for tritium breeding). Beryllium as a PFM has been used since 1990s in the high power fusion devices like JET tokamak ${ }^{[1]}$.

Lithium has strong oxygen getter activity and strong hydrogen retention ${ }^{[2]}$. Beryllium has the advantage of being a low-Z material so low risk of plasma contamination, non-reactive with hydrogenic isotopes and has good thermal conductivity ${ }^{[3,4]}$, however, its dust is toxic when ablating and expanding into the vacuum vessel of the reactor. Echols and Winfrey conducted computational work using the electrothermal plasma code ETFLOW in the ideal plasma regime for capillary discharge to simulate high heat flux deposition on materials and their ablative behavior ${ }^{[5]}$. They compared performance of beryllium and lithium at heat fluxes between 10 and $125 \mathrm{GW} / \mathrm{m}^{2}$ with capillary discharge currents between 9.5 to $76 \mathrm{kA}$. They reported the highest ablation for beryllium as compared to lithium ${ }^{[5]}$. The present study compares beryllium and lithium in the nonideal plasma regime for heat fluxes between 57 and 288 $\mathrm{GW} / \mathrm{m}^{2}$ with capillary discharge currents between 50 to $250 \mathrm{kA}$ over a $120 \mu$ s pulse length.

The advantages of beryllium are in its low $Z$ number, less fuel dilution and lower radiative power loss. Additionally, its high oxygen gettering ability, and the fact that it does not chemically sputter in hydrogen plasma and its low hydrogen inventory ${ }^{[3,4]}$ encourages many researchers to consider beryllium over other PFM ${ }^{[6]}$. Hassanein and Ehst compared the performance of Beryllium and graphite under hard disruption conditions expected in ITER by using the A*THERMAL computer code ${ }^{[3]}$; they found that both beryllium and graphite have advantages and disadvantages depending on the design, engineering and operation ${ }^{[3]}$. The very strong disruption in the next-step fusion reactors may need more loaded material instead of the current plasma facing materials of $\mathrm{C}, \mathrm{Be}$, and $\mathrm{W}$.

In 2007 ITER design review confirmed the selection of beryllium for the first-wall components based on its acceptable effect on plasma performance ${ }^{[6]}$, and the knowledge gained from the JET reactor that uses Be tiles.

Lithium as a low atomic number material has a high latent heat of evaporation, low melting temperature and not a serious source of impurities that induces a rise in
$\mathrm{Z}_{\mathrm{eff}}$, and several applications in lithium technologies such as Li-pellet injection ${ }^{[7]}$, as well as liquid surface and/ or capillary-pore limiters ${ }^{[8-10]}$, which are widely used in contemporary magnetic confinement devices. J. A. Snipes injected solid lithium and boron pellets into TFTR plasma to coat the graphite inner wall bumper limiter with a small amount of lower $\mathrm{Z}$ pellet material, which improved the plasma performance and the addition of a small amount of $\mathrm{Li}$ on a graphite target reduce the $\mathrm{C}$ sputtering yield ${ }^{[7]}$.

V. A. Evtikhin et al. have performed experiment with lithium CPS on the hydrogen plasma accelerator MK-200 where they found that in the vicinity of the CPS surface a dense protective layer of lithium plasma is formed, due to which a solid CPS structure will not be damaged under a short-term high thermal load ${ }^{[1]}$. Solid CPS filled with liquid lithium(CPS) as a plasma facing material has many advantages such as high resistance to radiation damage and thermal stresses, cracking, melting in steady state and during plasma transitions (disruptions and Edge Localized Modes "ELM"), and possess surface self-regeneration through surface tension forces, which are basically different from the solid material divertor concept

J. S. Hua et al. have performed first experiment of liquid lithium limiter on HT-7 tokamak and reported that the core electron temperature slightly increased, the particle confinement time increased by a factor of 2 , and a $20 \%$ increase in the energy confinement time ${ }^{[12]}$. After lithium coating, the hydrogen recycling decreased, and core electron temperature increased significantly by a factor of 2 . At the same time, after lithium coating, electron density of edge plasmas obviously decreased while electron temperature slightly increased ${ }^{[12]}$.

Improvement in plasma performance is noticed when coating the graphite inner wall bumper limiter with a small amount of Lithium and Boron which reduces the influx of carbon from the walls so a progress in the wall conditioning is achieved ${ }^{[13]}$.

Our purpose in this research is to judge the performance of $\mathrm{Li}$ and $\mathrm{Be}$ and evaluate their erosion rates. Electrothermal plasma capillary discharges have been used to simulate the high-heat flux deposition on materials to assess their erosion rates. The electrothermal plasma code ETFLOW, which is written for capillary discharges to predict the plasma parameters and erosion rates, simulates the highheat flux conditions similar to expected disruption events. It has been used in this study for simulated heat fluxes from as low as $\sim 50$ to as high as $\sim 290 \mathrm{GW} / \mathrm{m}^{2}$ with a reconnoitering of generating the beryllium and lithium plasmas up to the third ionization $\left(\mathrm{Be}^{+++}, \mathrm{Li}^{+++}\right)$. 


\section{Ideal and Non-ideal Plasma Models in the ETFLOW Computer Code}

Collisional processes in plasma determine its electrical conductivity from the electro-ion and electron-electron collisions, as well as contribution for electron-neutral atoms collisions. Collisional processes are ideal for lowdensity plasmas, however, if the density increases the collision between particles also increases and the mean energy of inter-particle interaction increases.

When the strong potential energy of the interacting particles exceeding their kinetic energies the ideal Spitzer model ${ }^{[14]}$ does not describe the plasma well, and hence there is need for the non-ideal model for high-density plasmas.

High density plasma with non ideal effects is recognized in many natural phenomena and devices, and in nature such as super dense plasma of white dwarfs, the sun and the deep layers of giant planets in the solar system; and in energy-related project devices like pulsed fusion, powerful MHD generators and rocket engines.

The parameter $\gamma=e^{2} n^{1 / 3} / 4 \pi \varepsilon_{0} k T_{e}$ defines the plasma to differentiate between ideality and non ideality based on the interaction between the charged particles, Where $\mathrm{n}$ is the sum of the electron and ion number densities, $\mathrm{k}$ is Boltzmann constant, $\varepsilon_{0}$ is permittivity of free space, and $\mathrm{T}_{\mathrm{e}}$ is the plasma electron temperature. This parameter defines plasma as ideal if $\gamma<<1$ and nonideal for $\gamma>1$.

The Coulomb logarithm is the main feature difference between the ideal and non-ideal plasma models and is given by $\ln (\Lambda)=\ln \left(1.23 \times 10^{7} T^{3 / 2} / n^{1 / 2} \bar{Z}^{-3 / 2}\right)$ for ideal plasmas, where $\mathrm{T}$ is the plasma kinetic temperature, $\mathrm{n}$ is the number density and $\bar{Z}$ is the average charge state. An exact analytical model for the Coulomb logarithm for non-ideal plasma has been derived by Zaghloul et al. ${ }^{[15,16]}$, which replaces the standard Coulomb logarithm and covers the range of ideal and nonideal plasmas and is given by $\ln (\Lambda)=\frac{\pi}{2} \sin (3 / 2 \Lambda)\left[1-\frac{2}{\pi}\left(\operatorname{Si}(3 / 2 \Lambda)+\frac{\operatorname{Ci}(3 / 2 \Lambda)}{\operatorname{Tan}(3 / 2 \Lambda)}\right)\right]$. Both models, ideal and nonideal Coulomb logarithms, are included in a special routine in the ETFLOW code of the capillary discharge, which simulates typical disruption high heat flux deposition, and calculates the plasma conductivity in the Joule heating term in the energy equation.

This model is effective in calculating the joule heating term in the energy equation for electrothermal plasmas, and covers the entire range from weakly to strongly nonideal ${ }^{[15,16]}$. The code also includes the Spitzer ideal model for comparison of the results between ideal and non-ideal plasma regimes.

\section{Results and Discussion}

Figure 1 shows the plasma pressure of beryllium at the capillary exit, indicating a peak pressure of $3.28 \times 10^{8} \mathrm{~N} /$ $\mathrm{m}^{2}(328 \mathrm{MPa})$ at $50 \mathrm{kA}$ to $1.16 \times 10^{9} \mathrm{~N} / \mathrm{m}^{2}(1158 \mathrm{MPa})$ at $250 \mathrm{kA}$. The pressure peaks at about $15 \mu \mathrm{s}$ after the peak of the discharge current as the discharge current is the source of Joule heating of the capillary arc, which emits radiant heat flux and initiates surface ablation and ejection of the particulates from the liner material, followed by dissociation and ionization, and hence the pressure buildup follows. At about $50 \mu$ s the pressure falls down slowly. Same trends are observed for lithium, as shown in Figure 2. A comparison between $\mathrm{Be}$ and $\mathrm{Li}$ peak pressures is illustrated in Figure 3, in which it is clear that lithium produces higher pressure at the capillary exit.

It is noticed that the increase in pressure is doubled with the increase in the current. For example, the difference between the exit pressure of $\mathrm{Li}$ and $\mathrm{Be}$ at 100 $\mathrm{kA}$ current is $5.000 \mathrm{E}+08$ while the difference between the exit pressure of $\mathrm{Li}$ and $\mathrm{Be}$ at $150 \mathrm{kA}$ current is $14.00 \mathrm{E}+08$, however the difference between the exit pressure of $\mathrm{Li}$ and $\mathrm{Be}$ at $200 \mathrm{kA}$ current is $23.000 \mathrm{E}+08$.

Time evolution of the plasma temperature of $\mathrm{Li}$ at the capillary exit is illustrated in Figure 4 for the tested range of peak discharge current 50-250 kA. As noticed the temperature increases continually until $10 \mu \mathrm{s}$ after that it falls down quickly until all temperatures of all currents have the same value $\left(12,000^{\circ} \mathrm{K}\right)$ at the end of discharging. The plasma temperature of Be has different behavior, it falls down slowly at the end of the discharge and each current has different temperature, for example at $50 \mathrm{kA}$ the plasma temperature at $120 \mu \mathrm{s}$ is $12,000^{\circ} \mathrm{K}$ while at 250 $\mathrm{kA}$ the plasma temperature at $120 \mu \mathrm{s}$ is $22,500^{\circ} \mathrm{K}$.

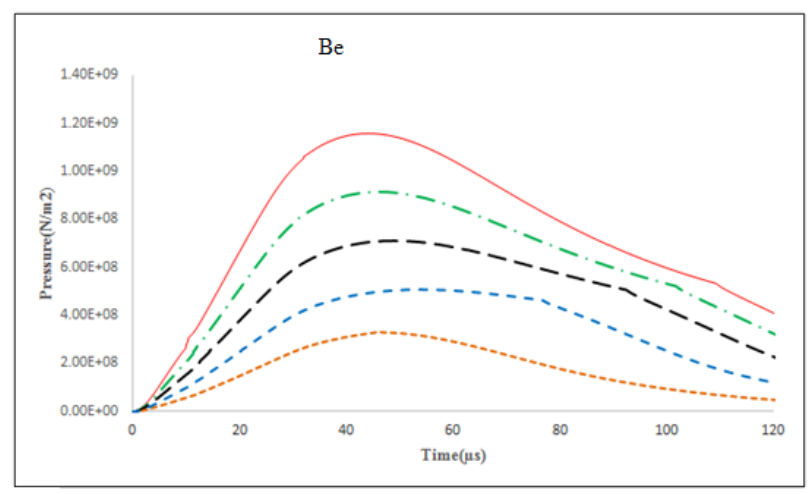

Figure 1. Plasma pressure of beryllium at the capillary exit 


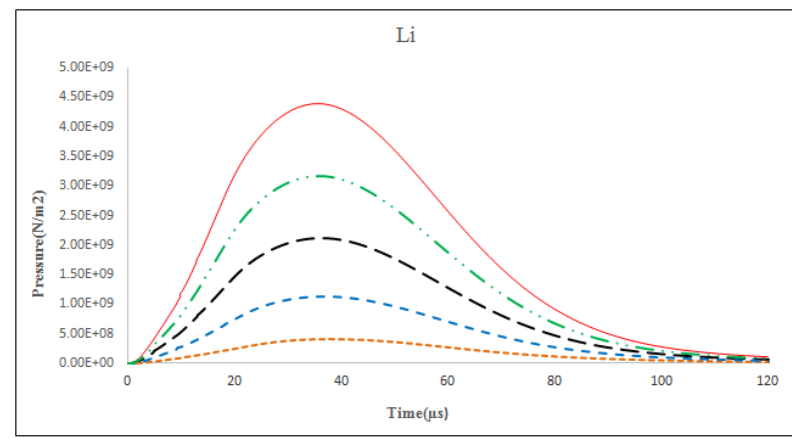

Figure 2. Plasma pressure of lithium at the capillary exit

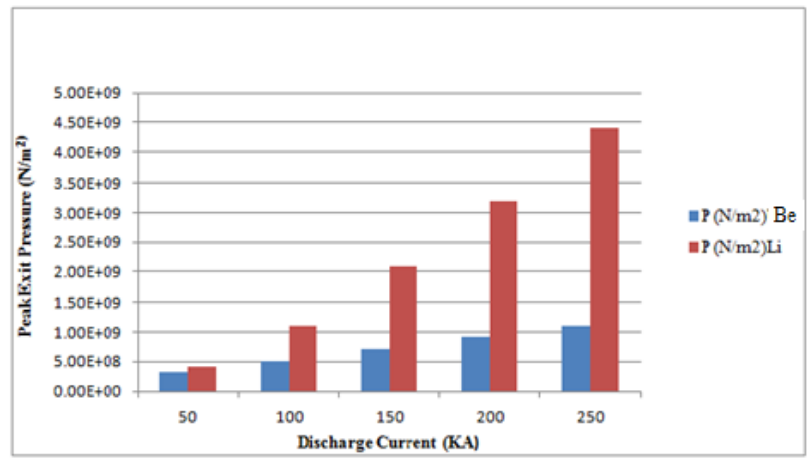

Figure 3. Comparison between $\mathrm{Be}$ and $\mathrm{Li}$ exit pressure at peak discharge currents

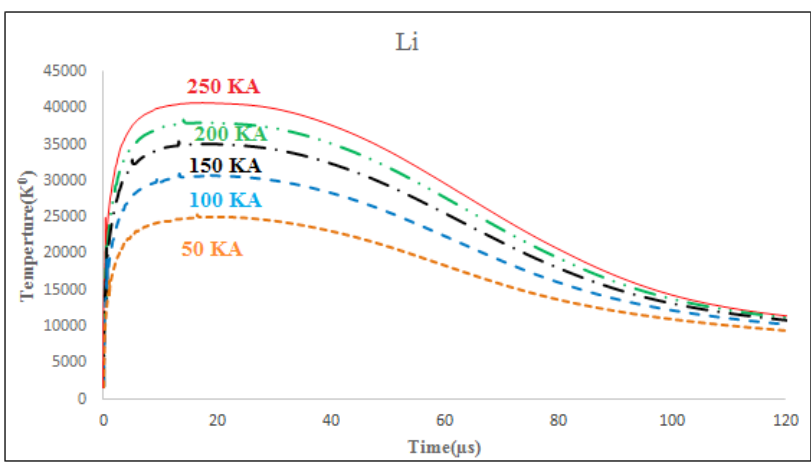

Figure 4. Plasma temperature versus time at the capillary exit for Li with currents up to $250 \mathrm{kA}$

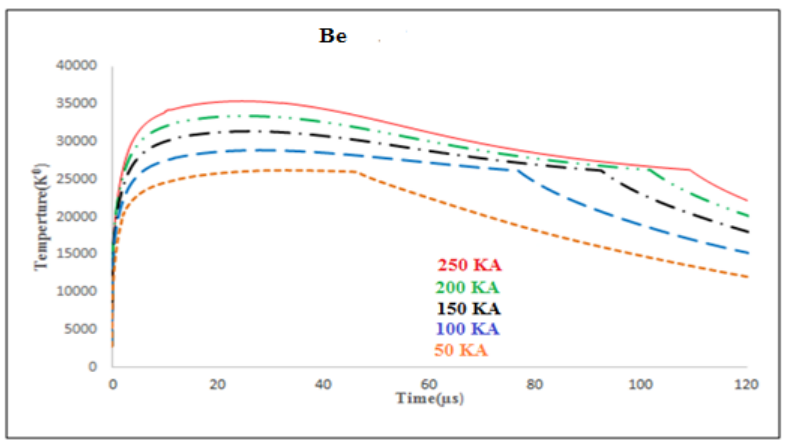

Figure 5. Plasma temperature versus time at the capillary exit for Be with currents up to $250 \mathrm{kA}$

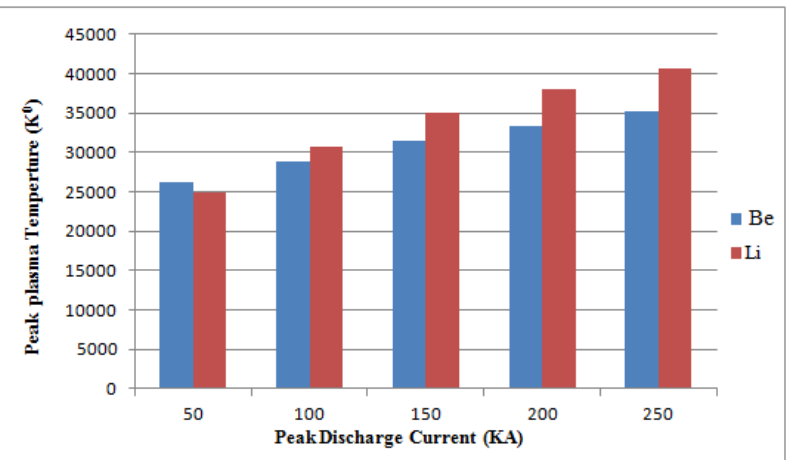

Figure 6. Comparison between plasma temperature for Be and $\mathrm{Li}$ at different peak currents

Figure 6 shows peak plasma temperatures of $\mathrm{Li}$ and $\mathrm{Be}$ with the peak of the discharge current. It is noticed that the temperature of $\mathrm{Be}$ is greater than that of $\mathrm{Li}$ at $50 \mathrm{kA}$ discharge current, which agrees with the ideal behavior. However, the non-ideal behavior starts to appear at 100 $\mathrm{kA}$ current where the temperature of $\mathrm{Li}$ is becoming greater than that of beryllium.

The heat flux drops to a minimum for all discharge currents at the end of the discharge time, as shown in Figure 7 for $\mathrm{Be}$ and Figure 8 for $\mathrm{Li}$, with values between 6.2-7.2 $\mathrm{GW} / \mathrm{m}^{2}$ for $50-250 \mathrm{kA}$, respectively.

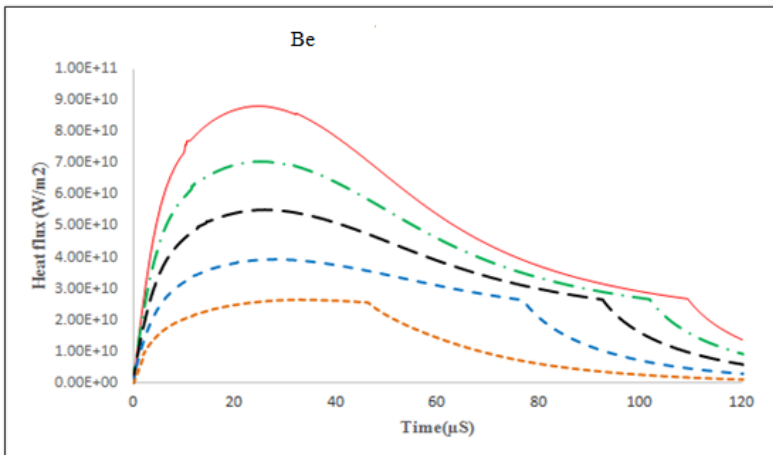

Figure 7. Heat flux versus time at the capillary exit when using $\mathrm{Be}$ as the liner

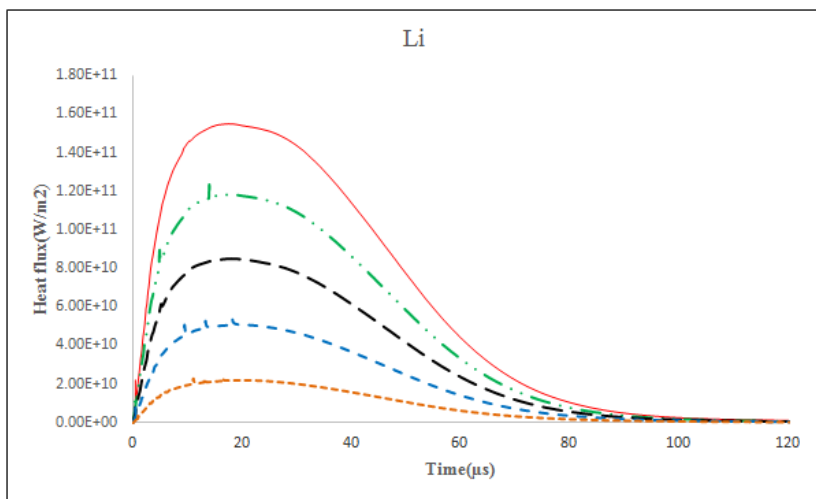

Figure 8. Heat flux versus time at the capillary exit when using $\mathrm{Li}$ as the liner 


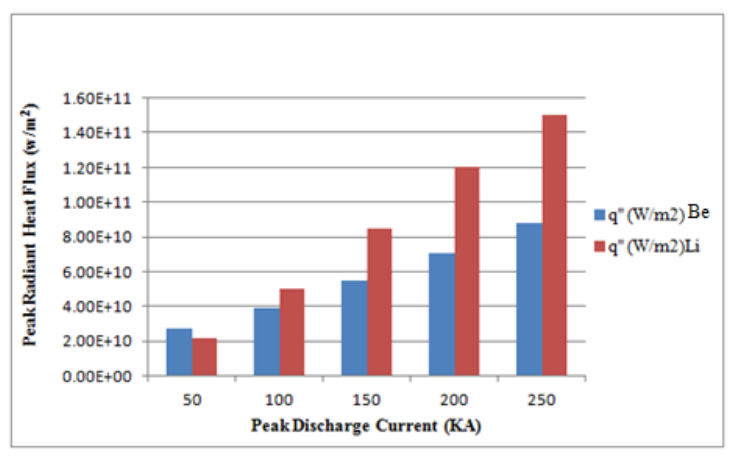

Figure 9. Heat flux versus peak current $\mathrm{Li}$ and $\mathrm{Be}$ at the capillary exit

Figure 9 shows the increase in the peak heat flux of $\mathrm{Li}$ and Be with the increase of the discharge current. Lithium shows increase in the heat flux more than Be except at 50 $\mathrm{kA}$ current where the heat flux of Be is greater than that of $\mathrm{Li}$, which in turn affects the total ablated mass as shown in Figure 10.

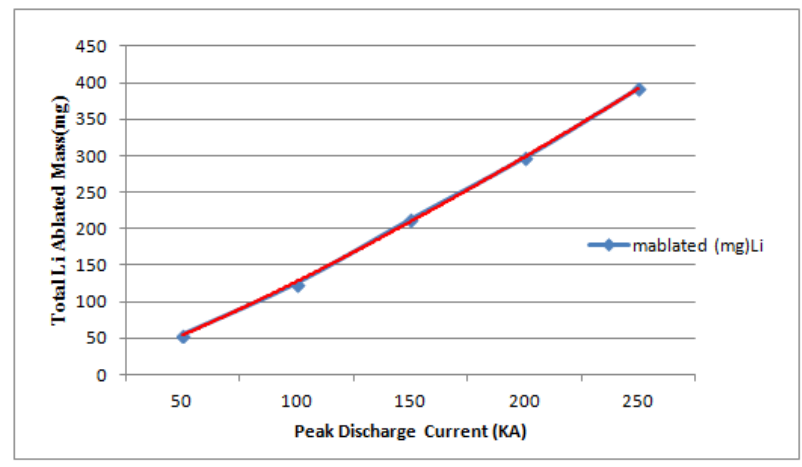

Figure 10. total ablated mass of Li versus peak discharge current

Figure 10 shows the total Li ablated mass released from the capillary for current values between 50 to 250 $\mathrm{kA}$. Total ablation for $50 \mathrm{kA}$ is $55 \mathrm{mg}$ and increases to $392 \mathrm{mg}$ for $250 \mathrm{kA}$. The power law is shown to be the best fitting with respect to the discharge current $\left(m_{\text {Li total ablated }(m g)}=5.47 I_{\text {peak }(k A)}^{1.22}\right)$ and agrees with the general scaling of plasma parameters ${ }^{[17]}$.

Mass ablation inside the source does not include any re-deposition or re-solidification and the total ablated mass is removed out of the capillary by the axial flow and the pressure effect.

Figure 11 shows the total Be ablated mass released from the capillary for current values between 50 to 250 $\mathrm{kA}$. Total ablation for $60.8 \mathrm{kA}$ is $55 \mathrm{mg}$ and increases to $225 \mathrm{mg}$ for $250 \mathrm{kA}$. A fit in a power law is preferable $\left(m_{\text {Be total ablated }(m g)}=61.131 I_{\text {peak }(k A)}^{0.815}\right)$ and is also correlating well to the general scaling of plasma parameters ${ }^{[17]}$.

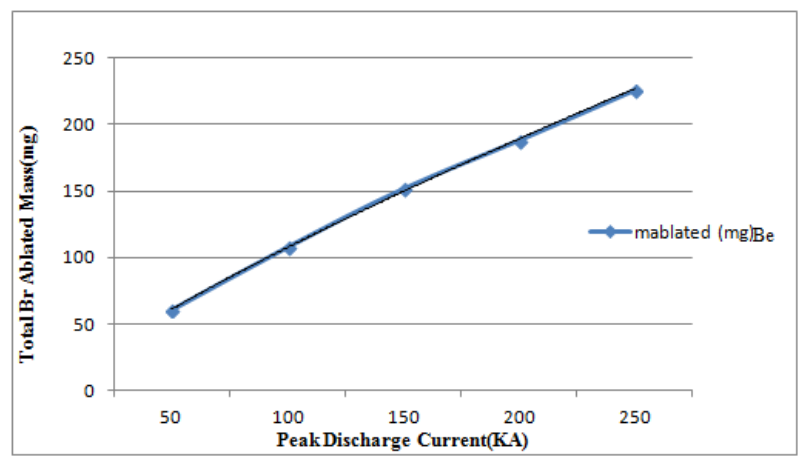

Figure 11. Beryllium total ablated mass versus peak discharge current

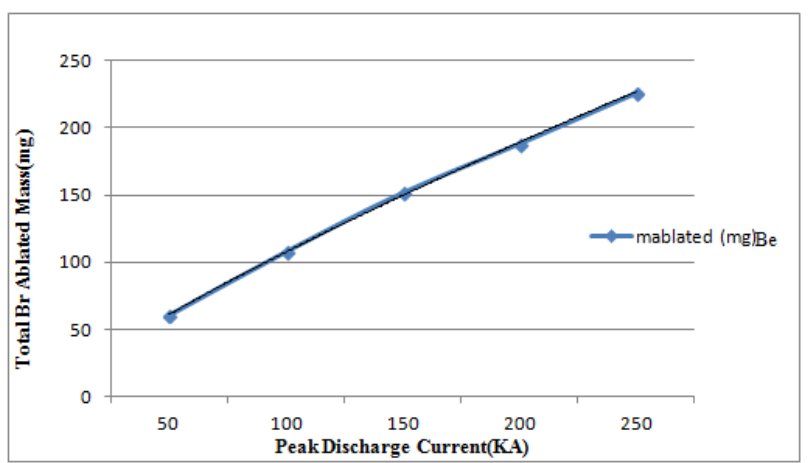

Figure 12. Total ablation of $\mathrm{Li}$ and Be versus peak discharge current

Figure 12 illustrates the total ablated mass of $\mathrm{Be}$ and $\mathrm{Li}$ versus the peak discharge current. It is noticed that at $50 \mathrm{kA}$ current the beryllium has the highest ablation, while lithium is the lowest which agrees with the results of J. R. Echols and A. L. Winfrey where the plasma is in ideal regime ${ }^{[5]}$. While the non ideal plasma regime is more clear at higher discharge currents of $100 \mathrm{kA}, 150$ $\mathrm{kA}, 200 \mathrm{kA}$ and $250 \mathrm{kA}$. Figure 13 shows the second and third ionization number densities of $\mathrm{Be}$ and $\mathrm{Li}$ versus peak discharge current, indicating initiation of third ionization when the discharge current exceeds $150 \mathrm{kA}$. The increase in third ionization species provides contamination inside the core of plasma in fusion reactors. Lithium generates third ionized species much higher than Be, especially at high currents but they recombine more rapidly.

Figure 14 shows the peak number densities of all lithium plasma constituents, electrons, ions (first, second and third ionizations), neutrals and the total number density. The lines of electron number density and the number density of first ionization are nearly congruent because the most of electrons comes from the first ionization, the total number densities from second and third ionization are small relative to the number density of the first ionization. As noticed while the pulse length is 
increased, the number density of individual particles stays constant (which is expected due to the balance between the ionization and recombination rates) except the second and third ionization which increase for the first $25 \mu$ s then drops more rapidly near the end of the discharge period as a result of the recombination processes. The life time of third ionization is almost half the life time of second ionization.

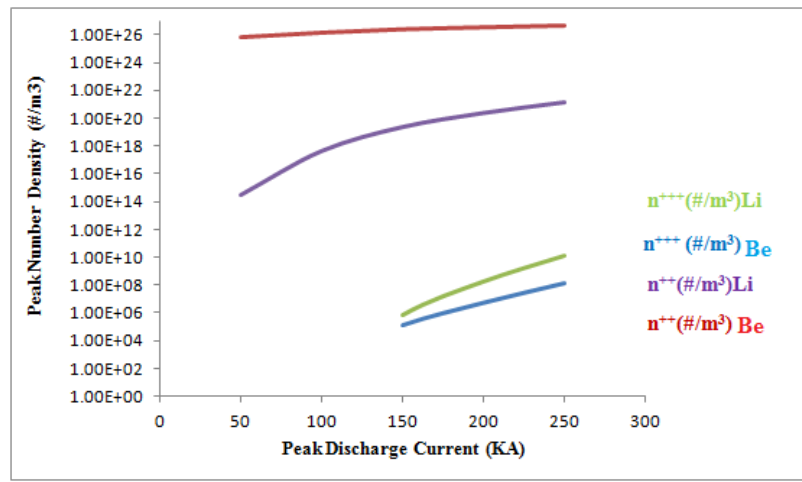

Figure 13. Second and third ionization number densities of $\mathrm{Be}$ and Li versus peak discharge current

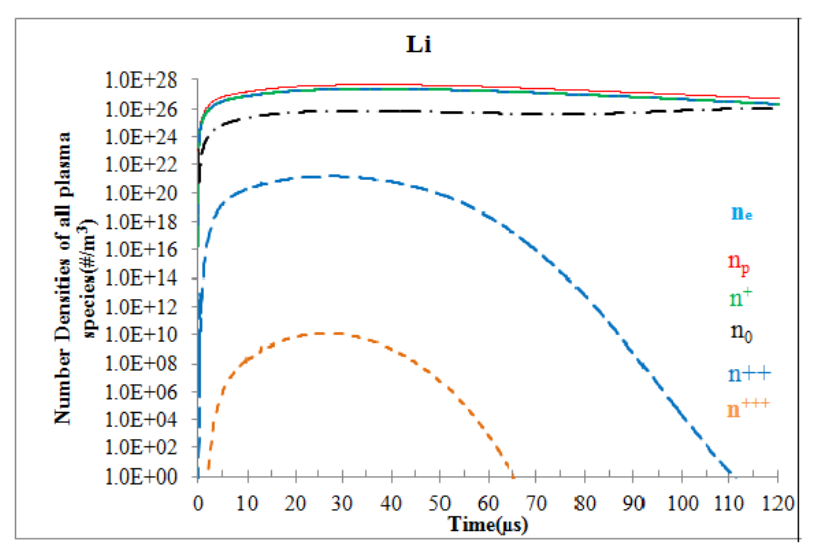

Figure 14. Number density of lithium ions compared to the total number density and the density of electrons

Figure 15 shows the peak number densities of all beryllium plasma constituents, electrons, ions (first, second and third ionizations), neutrals and the total number density. On contradiction with Li behavior, the number density of second ionization of Be stays unchanged with the increase of the pulse length as other individual particles, however the third ionization increases for the first $30 \mu$ s then slowly decays towards the end of the discharge period. The third ionization of Be is more consistent than the third ionization of $\mathrm{Li}$ which disappears completely at $65 \mu \mathrm{s}$.

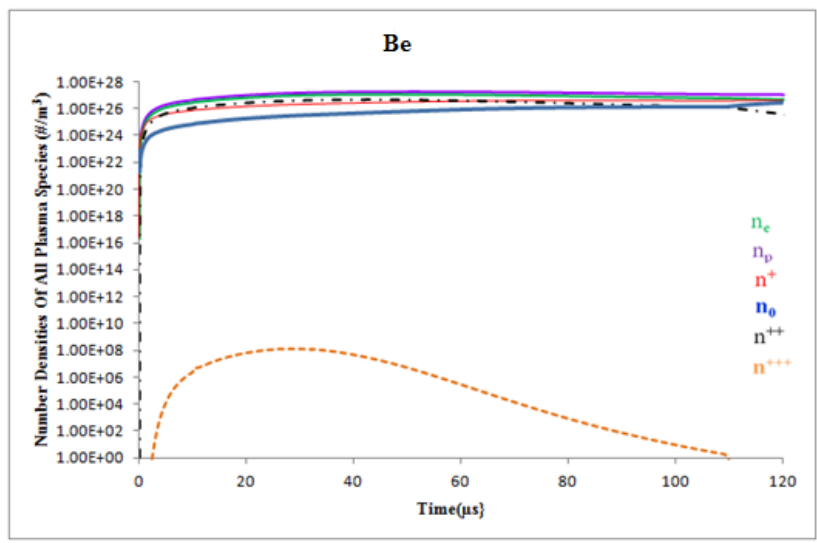

Figure 15. Number density of beryllium ions compared to the total number density and the density of electrons

Figure 16 shows the peak plasma-bulk velocities of $\mathrm{Li}$ and $\mathrm{Be}$ at the capillary exit versus the peak discharge current. As shown in the figure Li has the highest values of velocities which reaches $9000 \mathrm{~m} / \mathrm{s}$ at $250 \mathrm{kA}$ while the maximum velocity of Be at $250 \mathrm{kA}$ is about $6500 \mathrm{~m} / \mathrm{s}$ so many researchers used $\mathrm{Li}$ as pellet injection like the work of J. L. Terry et al. where they used injection of highspeed Li pellets to measure the internal magnetic field pitch on TFTR ${ }^{[18]}$.

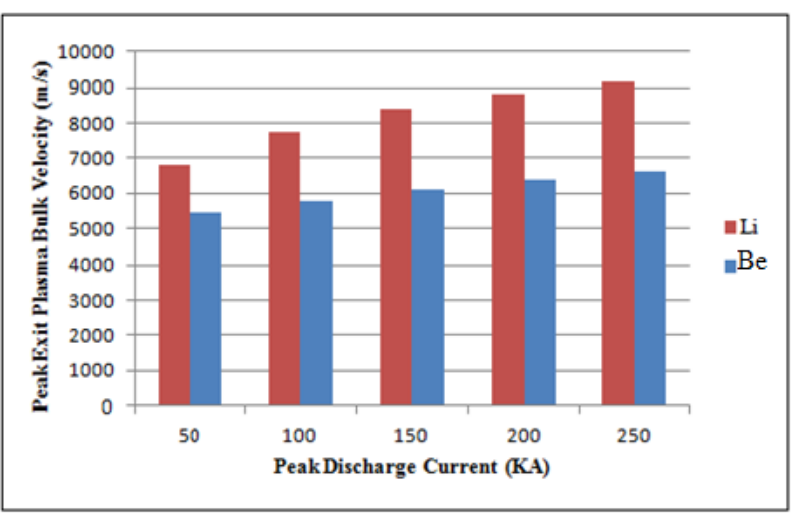

Figure 16. comparison between peak plasma bulk velocity at different peak discharge currents

Figure 17 shows the time evolution of the plasma bulk velocity at the last node (the capillary exit) for the 50 and $250 \mathrm{kA}$ discharge currents. At the initiation of the plasma formation, the velocity rises sharply to reach its peak at the peak of the discharge current. The higher current has faster rise as compared to lower current values as shown in the figure. The velocity decreases with the decrease in the magnitude of the discharge current and drops from its peak of $5077 \mathrm{~m} / \mathrm{s}$ for $50 \mathrm{kA}$ to $2964 \mathrm{~m} / \mathrm{s}$ at the end of the discharge at $120 \mu \mathrm{s}$. For the higher discharge current of $250 \mathrm{kA}$, the bulk velocity drops from its peak value of $6507 \mathrm{~m} / \mathrm{s}$ to $3811 \mathrm{~m} / \mathrm{s}$ at $120 \mu \mathrm{s}$. It concludes that the exit 
velocity at the end of the discharge cycle is in the range of 2900-3800 m/s for currents between 50 to $250 \mathrm{kA}$. Similar behavior can be seen in Figure 18 when beryllium is the liner material in the capillary.

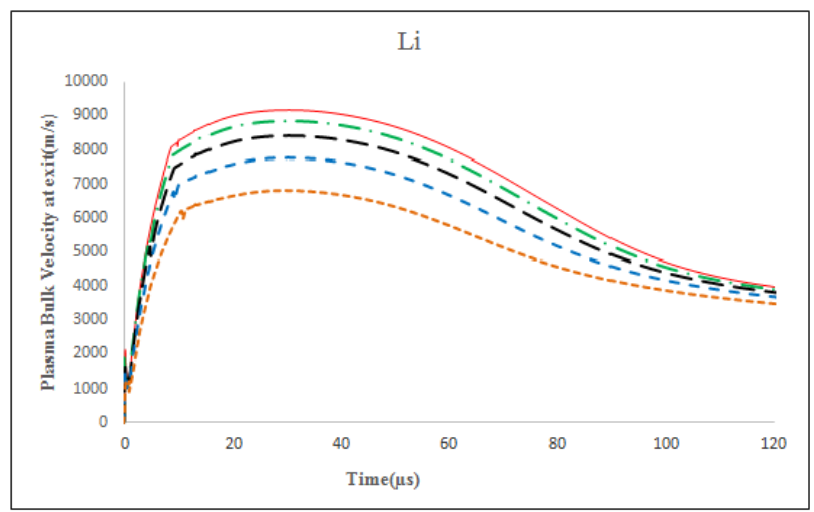

Figure 17. Plasma bulk velocity at source exit for lithium liner in the capillary

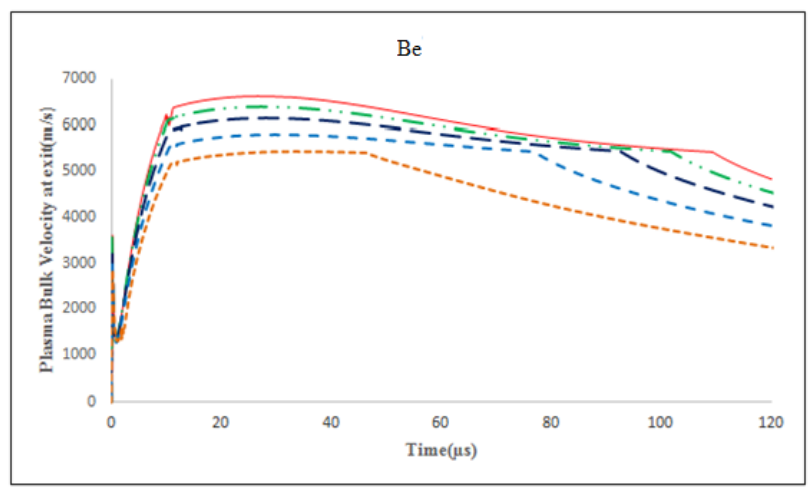

Figure 18. Plasma bulk velocity at source exit for beryllium liner in the capillary

\section{Conclusions}

A detailed study was performed to compare between Beryllium and Lithium as fusion materials for plasma-facing components in future fusion reactors. Computational experiments using ETFLOW code were conducted. Beryllium and lithium demonstrate lower ablation rates at all levels of tested heat fluxes between 10 and $125 \mathrm{GW} / \mathrm{m}^{2}$, however the ablation rates increase sharply from $125 \mathrm{GW} / \mathrm{m}^{2}$ and $288 \mathrm{GW} / \mathrm{m}^{2}$, especially $\mathrm{Li}$ which shows double ablation rates more than Be. Both $\mathrm{Li}$ and Be have a specific merits which nominate each of them to a fixed function inside the reactor that related to design, engineering, and operation are rather closely matched. The generation of third ionization is a particular behavior regarding using peak discharge currents of 50$250 \mathrm{kA}$, which produces heat fluxes from 57 to $288 \mathrm{GW} /$ $\mathrm{m}^{2}$. The generation of third ionization starts obviously from $150 \mathrm{kA}$ for both element and $\mathrm{Li}^{+++}$shows a small increasing than $\mathrm{Be}^{+++}$by two orders of magnitude at $250 \mathrm{kA}$. Plasma temperature varies from $25000 \mathrm{~K}$ for both elements to 35000 for Be and 40000 for $\mathrm{Li}$ at the lowest and highest heat fluxes, respectively. The high exit velocity of $\mathrm{Li}$ which arrives at $9000 \mathrm{~m} / \mathrm{s}$ nominates it to be used as a high-speed pellet injector inside the reactors for deep fueling. The generation of $\mathrm{Be}^{++}$which is nearly equals $\mathrm{Be}^{+}$is staying unchanged and does not suffer any recombination for a long time. Achieving higher ionizations in such high-density plasmas turns the plasma to behave weakly nonideal and hence the conductivity model of the non-ideal plasma was employed.

\section{References}

[1] Robert, W.C., Russell, P. Doerner, Jongik Won, 1997. Beryllium as the plasma-facing material in fusion energysystems - experiments, evaluation, and comparison with alternative materials, Fusion Engineering and Design. 37, 481-513.

[2] Asif, M., 2014. The HT-7 Team, Theoretical Calculation of Effective Ionic Charge with Lithium Limiter on HT-7 Tokamak, J Fusion Energ. 33, 444-448.

[3] Hassanein, A., Ehst., D.A., 1994. Beryllium and graphite performance in ITER during a disruption, Journal of Nuclear Materials. 212-215, 1272-1277.

[4] Federici, G., Skinner, C.H., Brooks, J.N., Coad, J.P., Grisolia, C., Haasz, A.A., Hassanein, A., Philipps, V., Pitcher, C.S., Roth, J., et al., 2001. Plasma-material interactions in current tokamaks and their implications for next step fusion, reactors Nuclear Fusion. Vol. 41, No. 12R.

[5] Echols, J.R., Winfrey, A.L., 2014. Ablation of Fusion Materials Exposed to High Heat Flux in an Electrothermal Plasma Discharge as a Simulation for Hard Disruption, J Fusion Energ. 33, 60-67. DOI: https://doi.org/10.1007/s10894-013-9639-4.

[6] Barabash, V., Eaton, R., Hirai, T., Kupriyanov, I., Nikolaev, G., Wang, Zh.H., Liu, X., Roedig, M., Linke, J., 2011. Summary of beryllium qualification activity for ITER first-wall applications, Phys. Scr. T145, 014007.

[7] Snipes, J.A., 1992. Wall conditioning with impurity pellet injection on TFTR, Journa of nuclear materials. 196-198, 686-691.

[8] Majeski, R., Jardin, S., Kaita, R., Gray, T., Marfuta, P., Spaleta, J., Timberlake, J., Zakharov, L.E., Ghassan Antar, R., Doerner et al., 2005. Nucl. Fusion. 45, 519-523.

[9] Mirnov, S.V., Azizov, E.A., Evtikhin, V.A., Lazarev, V.B., Lyublinski, I.E., Vertkov, A.V., Yu Prokhorov, 
D., 2006. Plasma Phys. Control. Fusion 48, 821.

[10] Apicella, M.L., Lazarev, V., Lyublinski, I., Mazzitelli, G., Mirnov, S., Vertkov, A., 2009. Lithium capillary porous system behavior as PFM in FTU tokamak experiments. Journal of Nuclear Materials. 386-388, 821-823.

[11] Evtikhin, V.A., Lyublinski, I.E., Vertkov, A.V., Azizov, E.A., Mirnov, S.V., Lazarev, V.B., Sotnikov, S.M., Safronov, V.M., Prokhorov, A.S., Korzhavin, V.M., June 2004. Main Directions and Recent Test Modeling Results of Lithium Capillary-Pore Systems as Plasma Facing Components, Plasma Science \& Technology. vo1.6, No.3.

[12] Hu, J.S., Zuo, G.Z., Li, J.G., Luo, N.C., Zakharov, L.E., Zhang, L., Zhang, W., Xu, P., 2010. Investigation of lithium as plasma facing materials on HT-7, J.S. Hu et al. / Fusion Engineering and Design. 85, 930-934.

[13] Ruzic, D.N., Shaheen, M., 1992. Wall conditioning with impurity pellet on TFTR, Journal of nuclear materials. 196-198, 686-691.

[14] Spitzer, L., Härm, R., Mar. 1953. Transport phenomena in a completely ionized gas. Phys. Rev. 89(5),
971-981.

[15] Zaghloul, M.R., Bourham, M.A., Doster, J.M., Powell, J.D., October 1999. On the Average Electron-Ion Momentum Transport Cross-Section in Ideal and Nonideal Plasmas. Physics Letters A. Vol. 262/1, pp. 86-89.

[16] Zaghloul, M.R., Bourham, M.A., Doster, J.M., April 2000. Energy-Averaged Electron-Ion Momentum Transport Cross-Section in the Born Approximation and Debye-Huckel Potential: Comparison with the Cut-Off Theory. Physics Letters A. Vol. 268/4-6, pp.375-381.

[17] Vergara, P.P., Gilligan, J., Winfrey, L., Bourham, M., 2015. Generalized Scaling Laws of Plasma Parameters in Electrothermal Plasma Sources for Fusion Disruption Erosion and Hypervelocity Launch Applications, IEEE Trans Plasma Sc. 43(10), 3645-3652 .

[18] Terry, J.L., Marmar, E.S., Howell, R.B., 1990. Measurement of internal magnetic field pitch using $\mathrm{Li}$ pellet injection on TFTR (invited), Review of Scientific Instruments. 61, 2908.

DOI: https://doi.org/10.1063/1.1141775. 